R.J. Marks II, J.F. Walkup and M.O. Hagler, "Methods of linear system characterization through response cataloging", Applied Optics, vol. 18, pp. 655-659 (1979). 


\title{
Methods of linear system characterization through response cataloging
}

\author{
Robert J. Marks II, John F. Walkup, and Marion O. Hagler
}

\begin{abstract}
Various methods of linear system characterization are presented. Each method requires cataloging the system response to a set of input stimuli. The continuum orthonormal basis set response method, in principle applicable to all linear systems, has as special cases point-spread function and frequency response superposition integral characterizations. The piecewise isoplanatic approximation, sampling theorem approach, and discrete basis set response methods are three schemes whereby restrictive assumptions are placed on the system and/or allowable input class. In each case, the number of system responses required for system definition is reduced to a countable number. Contrasting properties and applicability of the three schemes are discussed. Last, shift-invariant and spreadless systems are briefly reviewed in the context of requiring only one input-output relation for complete system characterization.
\end{abstract}

\section{Introduction}

In the treatment of linear (space-variant) systems, a knowledge of the system point-spread function (impulse response) is normally assumed.1,2 In practical situations, this relation can be determined in sampled form by probing the input plane with point sources and cataloging the corresponding system responses. If, however, the system is highly space-variant and the response changes rapidly over a small interval in the input plane, an enormous number of responses needs to be cataloged. In such a case, an alternate method of system characterization might be more appropriate. Linear system characterizations also play an important role in coherent linear processor synthesis. There have been efforts, for example, to synthesize space-variant processors through the multiplexing of sample pointspread functions either angularly in a volume hologram $^{3,4}$ or with phase coded reference beams. ${ }^{5}$ In both cases, an appeal to a space-variant sampling theorem characterization is made.

The purpose of this paper is to present and contrast a number of linear system characterizations. In the next section, we present the continuum orthonormal basis set response method, of which the familiar

R. J. Marks is with University of Washington, Department of Electrical Engineering, Seattle, Washington 98195; the other authors are with Texas Tech University, Department of Electrical Engineering, Lubbock, Texas 79409.

Received 28 September 1978.

0003-6935/79/050655-05\$00.50/0.

(C) 1979 Optical Society of America. point-spread function superposition integral is a special case. Reduction of the number of required input-output relations to a countable set is presented in Sec. III. Here, restrictive assumptions must be made on the system and/or allowable class of inputs. Systems requiring only a single input-output relationship are presented in Sec. IV. Section V contains some concluding remarks. For clarity of presentation, analysis will be performed in one dimension. Extension to two or more dimensions is straightforward.

We model the system process by the operator $S[\cdot]$ (Refs. 6, 7):

$$
g(x)=S[u(y)] .
$$

Here, $g(x)$ is the system output corresponding to an input $u(y) .8$ Henceforth, we restrict attention to the case where $S[\cdot]$ is linear:

$$
S\left[a u_{1}(y)+b u_{2}(y)\right]=a S\left[u_{1}(y)\right]+b S\left[u_{2}(y)\right],
$$

where $a$ and $b$ are $y$-independent constants.

\section{Continuum Orthonormal Basis Set Response}

A continuum set, $\left\{\psi_{x}(\xi)\right\}$, is said to be orthonormal and complete if ${ }^{7,9}$

$$
\int_{-\infty}^{\infty} \psi_{x}(\xi) \bar{\psi}_{y}(\xi) d \xi=\delta(x-y),
$$

where $\delta(\cdot)$ is the Dirac delta, and the overbar denotes complex conjugate. A signal $u(y)$ can be expressed in terms of this set by

$$
u(y)=\int_{-\infty}^{\infty} \alpha(\xi) \psi_{y}(\xi) d \xi
$$

where 


$$
\alpha(\xi)=\int_{-\infty}^{\infty} u(y) \bar{\psi}_{y}(\xi) d y .
$$

The proof follows straightforwardly from the sifting property of the Dirac delta. ${ }^{6}$

Substituting Eq. (2) into the system characterization in Eq. (1) and taking advantage of the operator's linearity give

$$
g(x)=\int_{-\infty}^{\infty} \alpha(\xi) S\left[\psi_{y}(\xi)\right] d \xi .
$$

Thus, our system is characterized by knowledge of the system's responses to each element in the continuum basis set. For a given input, one simply computes the corresponding $\alpha(\xi)$ followed by evaluation of Eq. (4).

\section{A. Line-Spread Function}

Historically, the most popular choice of a continuum orthonormal basis set is the Dirac delta: $\psi_{y}(\xi)=\delta(y-$ $\xi)$. Here, the functional coefficient $\alpha(\cdot)$ is identical to the input value at $y=\xi$. The system response is the familiar line-spread function: $h(x-\xi ; \xi)=S[\delta(y-\xi)]$. We here have used the Lohmann-Paris line-spread function notation. ${ }^{10,11}$ For the Dirac delta basis set, the system characterization in Eq. (4) becomes the familiar superposition integral: ${ }^{6}$

$$
g(x)=\int_{-\infty}^{\infty} u(\xi) h(x-\xi ; \xi) d \xi .
$$

\section{B. Frequency Response}

An alternate choice of a continuum basis set is the complex exponential: $\psi_{\nu}(\xi)=\exp (j 2 \pi \nu \xi)$. Here, $\nu$ is used instead of $y$ due to the obvious frequency personality of the variable. For this set, the functional coefficient is recognized as the Fourier transform of the input:

$$
\alpha(\nu)=\int_{-\infty}^{\infty} u(\xi) \exp (-j 2 \pi \nu \xi) d \xi=\mathscr{T}_{\xi}[u(\xi)]=U(\nu) .
$$

Borrowing from the sister electrical sciences, we shall call the system response to the complex exponential the frequency response:

$$
k(x-c \nu ; \nu)=S[\exp (j 2 \pi \nu \xi)] .
$$

This notation is analogous to that used for the Lohmann-Paris line-spread function. The constant $c$ merely retains dimensional consistency between the spatial variable $x$ and the frequency variable $\nu$. The input-output relation in this case is

$$
g(x)=\int_{-\infty}^{\infty} U(\nu) k(x-c \nu ; \nu) d \nu .
$$

Comparing with the superposition integral (Eq. (5)], we conclude from the power theorem ${ }^{12}$ applied to the spatial-frequency variables $(\xi, \nu)$ that

$$
k(x-c \nu ; \nu)=\int_{-\infty}^{\infty} h(x-\xi ; \xi) \exp (j 2 \pi \nu \xi) d \xi .
$$

The line-spread function and frequency response are thus related by a Fourier transform.

The frequency response characterization has an interesting physical interpretation. Instead of cataloging the system response to various input point sources, we here are cataloging the system response to a continuum number of tilted input plane waves. Thus instead of decomposing the input into a number of point source Huygen's wavelets, we are characterizing the input by its angular spectrum. ${ }^{6}$

Frequency response system characterization has been used to widen the class of operations achievable by 1-D coherent processors. ${ }^{13-16}$

\section{Discussion}

The point-spread function and frequency response lend themselves to an intuitive and physically realizable method of system characterization. They also are coupled by a Fourier duality. There do, of course, exist alternate continuum basis sets. Other basis sets, however, do not seem to lend themselves to easy implementation or intuitive interpretation. This is not to say, however, that better continuum basis sets do not exist for specific applications.

\section{Countable Discrete Characterizations}

By placing certain limiting assumptions on the system and/or input, it is possible to reduce the required number of cataloged input-output relations to a countable number. In this section, three such basic methods are reviewed and contrasted.

\section{A. Piecewise Isoplanatic Approximation}

The concept of the isoplanatic patch is well established with regard to imaging systems. ${ }^{6,17,18}$ Marks and $\mathrm{Krile}^{19}$ give a more extensive treatment with regard to general linear systems.

The basic assumption of the piecewise isoplanatic approximation (PIA) is that the system impulse response varies slowly as a Dirac delta explores an input interval $l_{n} \leq \xi \leq u_{n}$. In this case, the system output can be approximated by

$$
g(x) \simeq \sum_{n} \int_{l_{n}}^{u_{n}} u(\xi) h\left(x-\xi ; \xi_{n}\right) d \xi .
$$

The limits $\left(l_{n}, u_{n}\right)$ describe the boundaries of the $n$th isoplanatic patch. The point $\xi_{n}$ is chosen to lie somewhere within the $n$th patch. To assure no patch overlap, we further require that $u_{n}=l_{n+1}$.

The goodness of the approximation of the PIA is a relatively difficult quantity to measure in general. From the specific examples given in Ref. 19, however, we conclude that neither pointwise nor rms convergence can generally be assumed.

Note, however, that we have reduced the required number of input-output relations to a countable set. To apply the PIA, we need knowledge of only one sample impulse response per isoplanatic patch. Lohmann and Paris ${ }^{10}$ have offered a definition of the isoplanatic patch, which is wholly dependent on the system impulse response (line-spread function). We thus conclude that the PIA is essentially an input-independent method of linear system characterization.

As is the case with all the characterizations presented in this paper, the PIA has a Fourier dual. Here, the input spectrum is divided into frequency-invariant patches, and we make the approximation 


$$
g(x) \simeq \sum_{n} \int_{l_{n}}^{\hat{u}_{n}} U(\nu) k\left(x-c \nu ; \nu_{n}\right) d \nu,
$$

where $\hat{l}_{n}$ and $\hat{u}_{n}$ describe the patch's endpoints, and $\hat{l}_{n}$ $\leq \nu_{n} \leq \hat{u}_{n}=\hat{l}_{n+1}$. The sample frequency responses are obtained by cataloging the system response to a countable number of tilted plane wave inputs. Here again, the method of characterization is primarily input-independent.

\section{B. Sampling Theorem Characterizations}

Classical Whittaker-Shannon sampling theory is applicable to system characterization when certain bandlimited constraints are placed on the system input and line-spread function. For a detailed treatment with Fourier duals, see Refs. 16, 20, and 21.

A signal $u(y)$ is said to be bandlimited if its Fourier transform $U(\nu)$ has compact support, i.e., $U(\nu) \equiv 0$ for $|\nu|>W_{u}$, where $W_{u}$ is some finite number. Similarly, we say that a linear system described by the impulse response function $h(x ; \xi)$ is variation-limited if its Fourier transform with respect to $\xi$ has compact support. In other words, if $H_{\xi}(x ; \nu)$ is the Fourier transform of $h(x ; \xi)$, then $H_{\xi}(x ; \nu) \equiv 0$ for $|\nu|>W_{\nu}$ for all $x$, where $W_{\nu}$ is finite. Then, for a variation-limited system with a bandlimited input, the input-output relationship can be written as

$$
g(x)=\sum_{n} u\left(\xi_{n}\right) h\left(x-\xi_{n} ; \xi_{n}\right) * \sin c 2 W_{s} x,
$$

where $*$ denotes convolution, $\operatorname{sinc} x=\sin \pi x / \pi x, W_{s}=$ $W_{u}+W_{\nu}$, and $\xi_{n}=n / 2 W_{s}$. Note that the sampling rate $2 W_{s}$ is dictated by both the system and the input. As with the PIA, however, we only require the countable set $\left\{h\left(x ; \xi_{n}\right)\right\}$ for complete system characterization.

A second sampling theorem requires $h(x-\xi ; \xi)$ to be bandlimited in $\xi$ for all $x$. In this case

$$
g(x)=\frac{1}{2 W_{d}} \sum_{n} u\left(\xi_{n}\right) h\left(x-\xi_{n} ; \xi_{n}\right),
$$

where, now, $\xi_{n}=n / 2 W_{d}$. The minimum required sampling rate $2 W_{d}$ is equal to the sum of the input bandwidth $2 W_{u}$ and the supremum support of the Fourier transform of $h(x-\xi ; \xi)$ in $\xi$. The same set $\left\{h\left(x, \xi_{n}\right)\right\}$ is here required for system characterization. ${ }^{22}$

In both sampling theorems, desired sampling rates are dictated by both the input and the system. These characterizations are thus both input- and systemdependent.

\section{Discrete Basis Set Response}

Here we describe a method of linear system characterization, which places a constraint only on the allowable input class. ${ }^{4,16}$ Let $\left\{\phi_{n}(y)\right\}$ denote a complete orthonormal basis set dense in a signal class $A$. $A$ is assumed to be contained in the class of finite energy signals. ${ }^{23}$ Then, for all $u(y)$ in $A$, we can write ${ }^{24}$

$$
u(y)=\sum_{n} \alpha_{n} \phi_{n}(y),
$$

where $\alpha_{n}$ denotes the inner product

$$
\alpha_{n}=\int_{-\infty}^{\infty} u(y) \bar{\phi}_{n}(y) d y .
$$

Substituting the expansion into Eq. (1) and taking advantage of the operator's linearity give

$$
g(x)=\sum_{n} \alpha_{n} S\left[\phi_{n}(y)\right]
$$

From this relation we conclude that the system is completely characterized for the signal class $A$ by knowledge of its response to each element in the basis set. Note that the members of the response set $\left\{S\left[\phi_{n}(y)\right]\right\}$ need not necessarily be orthogonal. ${ }^{25}$ This scheme can, of course, be generalized by only requiring $\left\{\phi_{n}(y)\right\}$ to be a complete (not necessarily orthogonal) basis set in $A$. Note that no restrictions (except linearity) have been placed on the system.

An obvious choice for $\phi_{n}(y)$ is the set of eigenfunctions resulting from solution of the corresponding integral equation from Eq. (5). ${ }^{26-28}$ The fabrication of these eigenfunctions to input into the system, however, would in general be an extremely difficult undertaking and furthermore would require $a$ priori knowledge of the system impulse response. We will thus focus attention solely on the input class.

One common signal class consists of all finite energy bandlimited signals with bandwidth less than $2 W$. That is, if $u(y) \epsilon A$, then

$$
u(y)=\int_{-W}^{W} U(\nu) \exp (j 2 \pi \nu y) d \nu .
$$

Such signals can be expressed via the conventional cardinal series expansion ${ }^{6}$

$$
u(y)=\sum_{n} u\left(y_{n}\right) \operatorname{sinc} 2 W\left(y-y_{n}\right),
$$

where $y_{n}=n / 2 W$. We thus conclude that a linear system is characterized for this class of inputs if we have knowledge of $\left\{S\left[\operatorname{sinc} 2 W\left(y-y_{n}\right)\right]\right\}$ for all $n$. The system output is then found simply by weighting these responses by the input's sample values:

$$
g(x)=\sum_{n} u\left(y_{n}\right) S\left[\operatorname{sinc} 2 W\left(y-y_{n}\right)\right] .
$$

The input sinc basis set is one which can be straightforwardly generated by coherent optical means.

An alternate input signal class consists of finite energy signals of finite spatial width. We here consider the case where the input is identically zero outside of the interval $|y| \leq a$. The input can then be expressed in the Fourier series

$$
u(y)=\frac{1}{2 a} \sum_{n} U\left(\nu_{n}\right) \exp \left(j 2 \pi \nu_{n} y\right) \text { rect }\left(\frac{y}{2 a}\right),
$$

where $\nu_{n}=n / 2 a$ and

$$
\operatorname{rect}(x)=\left\{\begin{array}{l}
1 ;|x| \leq 1 / 2 \\
0 ;|x|>1 / 2 .
\end{array}\right.
$$

For this signal class, we can characterize the system by cataloging the responses

$$
S\left[\exp \left(j 2 \pi \nu_{n} y\right) \operatorname{rect}(y / 2 a)\right] \text {. }
$$

These elements can be easily generated by probing the system input with appropriately tilted plane waves 
through a rectangular aperture. The system output is gained by weighting these responses by the sample values of the input spectrum:

$$
g(x)=\frac{1}{2 a} \sum_{n} U\left(v_{n}\right) S\left[\exp \left(j 2 \pi v_{n} y\right) \operatorname{rect}\left(\frac{y}{2 a}\right)\right] .
$$

\section{Discussion}

In this section we have presented and reviewed three methods of linear system characterization with contrasting properties. In each case, the number of input-output relations required for characterization is countable.

The PIA is a characterization scheme which is only system-dependent. Sampling theorem representation, on the other hand, requires limiting assumptions on both the system and input. The third method requires cataloging the system response to each element in a basis set and was shown to be only input-dependent.

\section{Single Response Characterizations}

To complete our discussion, we now briefly review three restrictive system assumptions that allow system characterization by knowledge of only one system response.

By far the most common linear system assumption is that of shift-invariance. A system is said to be space-invariant (isoplanatic) if $h(x-\xi ; \xi) \rightarrow h(x-\xi)$. In this case the superposition integral in Eq. (6) becomes a convolution integral. Only a single impulse response $h(x)$ is required to completely define a linear spaceinvariant system. Many optical systems and, in particular, imaging systems are thus modeled. ${ }^{29,30}$

In a Fourier dual sense, we can call a system frequency-invariant ${ }^{31}$ if $k(x-c \nu ; \nu) \rightarrow k(x-c \nu)$. Thus, the only system response required for system definition is $k(x)$. This corresponds to cataloging the system response to a single normally incident input unit amplitude plane wave. A second response, however, may be required to determine the $c$ parameter. One can easily show that a linear system cannot be simultaneously space- and frequency-invariant.

An example of a frequency-invariant system is the common thin lens Fourier transformer with an inputoutput relationship of ${ }^{6}$

$$
g(x)=\int_{-\infty}^{\infty} u(\xi) \exp \left(-j 2 \pi x \frac{\xi}{\lambda f}\right) d \xi,
$$

where $f$ is the focal length of the lens, and $\lambda$ is the wavelength of the spatially coherent illumination. The corresponding frequency response is $k(x-c \nu)=\lambda f \delta(x$ $-\lambda f \nu)$. Note that the constant $c=\lambda f$ is here specified by system parameters.

One final linear system class that can also be considered as a dual of the shift-invariant system is the "spreadless" system ${ }^{1,27}$ with the input-output relationship defined by $g(x)=u(x) t(x)$. A simple mask with transmittance $t(x)$ is an example of such a system. The temporal analog of the spreadless system is the linear "memoryless" system. Like the frequency-invariant system, the spreadless system can obviously by characterized by its iesponse to a single normally inci- dent plane wave. The corresponding system response is $t(x)$.

The shift-invariant and spreadless systems impose severe limitations on applicable linear system classes. They do, however, require only one input-output relationship for complete characterization.

\section{v. Conclusion}

We have presented a number of methods by which a linear system can be characterized by cataloging its response to a set of input stimuli. These methods can either be used in the analysis or synthesis of linear coherent systems. In the latter case, we require a method of storing the system responses in such a manner that they are able to be appropriately accessed by an arbitrary input.

The best characterization method for specific application is determined by the system and/or allowable class of input. For example, a highly space-variant system would best be characterized by limiting the input class and using the discrete orthonormal basis set response method. If, on the other hand, our system is quasiisoplanatic (quasi-space-invariant), application of the PIA is more appropriate. In this case, no restrictive assumptions are made on the input.

The authors express their appreciation to Joseph Goodman and the reviewers for their excellent comments and suggestions. This work was supported in part by AFOSR grant 75-2855.

\section{References}

1. H. J. Butterweck, J. Opt. Soc. Am. 67, 60 (1977).

2. A. Papoulis, Systems and Transforms with Applications in Optics (McGraw-Hill, New York, 1968).

3. L. M. Deen, J. F. Walkup, and M. O. Hagler, Appl. Opt. 14, 2438 (1975).

4. R. J. Marks II, J. F. Walkup, and M. O. Hagler, "Volume Hologram Representation of Space-Variant Systems," in Applications of Holography and Optical Data Processing, E. Marom, A. A. Friesem, and E. Wiener-Aunear, Eds. (Pergamon, Oxford, 1977).

5. T. F. Krile, R. J. Marks II, J. F. Walkup, and M. O. Hagler, Appl. Opt. 16, 3132 (1977).

6. J. W. Goodman, Introduction to Fourier Optics (McGraw-Hill, New York, 1968).

7. J. D. Gaskill, Linear Systems, Fourier Transforms and Optics (Wiley, New York, 1978).

8. Here, $u(y)$ is a function of the input variable $y$. The dummy variable $\xi$, which occurs throughout this paper, also refers to the input variable. The operator $S[\cdot]$ maps the input space into the output space denoted by the variable $x$.

9. A. S. Davydov, Quantum Mechanics (Neo Press, Peaks Island, Maine, 1966).

10. A. W. Lohmann and D. P. Paris, J. Opt. Soc. Am. 55, 1007 (1965).

11. R. J. Marks II, J. F. Walkup, and M. O. Hagler, Appl. Opt. 15, 2289 (1976).

12. R. N. Bracewell, The Fourier Transform and Its Applications (McGraw-Hill, New York, 1965).

13. J. W. Goodman, P. Kellman, and E. W. Hansen, Appl. Opt. 16, 733 (1977).

14. R. J. Marks II, J. F. Walkup, M. O. Hagler, and T. F. Krile, Appl. Opt. 16, 739 (1977). 
15. R. J. Marks II, J. F. Walkup, and C. A. Irby, J. Opt. Soc. Am. 76, 1423A (1977).

16. R. J. Marks II, "Space-Variant Coherent Optical Processing," Ph.D. Thesis, Texas Tech. U., Lubbock, Texas (December 1977).

17. P. Elias, D. S. Grey, and D. Z. Robinson, J. Opt. Soc. Am. 42, 127 (1952).

18. P. B. Fellget and E. H. Linfoot, Philos. Trans. R. Soc. London Ser. A: 247, 369 (1955).

19. R. J. Marks II and T. F. Krile, Appl. Opt. 15, 2241 (1976).

20. R. J. Marks II, J. F. Walkup, and M. O. Hagler, J. Opt. Soc. 66, 918 (1976).

21. R. J. Marks II, J. F. Walkup, and M. O. Hagler, IEEE Trans. Circuits Syst. CAS-25, 228 (1978).

22. There also exist sampling theorem characterizations where a Dirac delta sweeps down the input plane and a sample array records the corresponding output at discrete sample points. ${ }^{16,21}$ This output sampling method deviates somewhat from our idea of cataloging system responses and will thus not be considered here.
23. In analysis, the class of (Lebegue measurable) finite energy signals is equivalent to $L_{2}(-\infty, \infty)$. A signal $u(y)$ is contained in this class if $^{24}$

$$
\int_{-\infty}^{\infty}|u(y)|^{2} d y<\infty .
$$

24. D. G. Luenberger, Optimization by Vector Space Methods (Wiley, New York, 1969), p. 58.

25. For example, consider the Laplace transform impulse response $h(x-\xi ; \xi)=\exp (-\xi x)$. Let $A$ denote all finite energy $L_{2}$ signals on the interval $[-1 / 2,1 / 2]$. Using Fourier series, we choose $\phi_{n}(y)$ $=\exp [-j 2 \pi n y)$. Then, $S\left[\phi_{n}(y)\right]=2 \sinh 1 / 2(x+j 2 \pi n) /(x+$ $j 2 \pi n)$. This response set is not orthogonal.

26. G. T. deFrancia, J. Opt. Soc. Am. 59, 799 (1969).

27. F. Gori, J. Opt. Soc. Am. 64, 1237 (1974).

28. B. Saleh, J. Opt. Soc. Am. 67, 71 (1977).

29. M. H. Peyrovian and A. A. Sawchuk, Appl. Opt. 17, 600 (1978).

30. E. J. Leith, Proc. IEEE 65, 18 (1977).

31. W. T. Rhodes and J. Florence, Appl. Opt. 15, 3073 (1976).
Meetings Calendar continued from page 640

1979

May

17-19 Trends and Applications Symp., NBS, Gaithersburg $S$. Watkins, B212 Technol. Bldg., NBS, Washington, D.C. 20234

21-25 1st Australasian Landsat Conf., Sydney J. Davies, P.O. Box 136, N. Ryde, NSW, 2113, Australia

21-25 Laser Safety course, Washington, D.C. Laser Inst. of Am., P.O. Box 9000, Waco, Tex. 76710

21-25 Fast Ion Transport in Solids-Electrolytes and Electrodes, internat. conf., Lake Geneva, Wisc. P. Vashishta, Solid State Sci. Div., ANL, Argonne, Ill. 60439

SPIE Huntsville Electro-Optical Tech. Symp. and Workshop, Von Braun Civic Ctr., Huntsville, Ala. SPIE, P.O. Box 10, Bellingham, Wash. 98225

23-24 Practical Application of Lasers in Manufacturing course Dearborn, Mich. Coherent, Inc., 3210 Porter Dr., P.O. Box 10321, Palo Alto, Calif. 94303

OSA Los Alamos Chapter, Los Alamos $V . K$ Viswanathan, LASL, P.O. Box 1663, Los Alamos, N.M. 87545

27-1 June 7th Canadian Congress of Applied Mechanics, U. of Sherbrooke N. Galanis, Faculty of Appl. Sci., U. of Sherbrooke, Sherbrooke, P.Q. J1K $2 R 1$

30-1 June IEEE/OSA Conference on Laser Engineering and Applications, Washington Hilton Hotel J. W. Quinn, OSA, 2000 L St. N.W., Washington, D.C. 20036

\section{June}

? AAS Meeting, Wellesley, Mass. L. W. Frederick, P.O. Box 3818, Univ. Station, Charlottesville, Va. 22903

4-8 Laser Optics course, Dallas Laser Inst. of Am., P.O. Box 9000, Waco, Tex. 76710

4-8 Quality Control for Photographic Processing, Graphic Arts Res. Ctr., RIT W. D. Siegfried, Coll. of Graphic Arts and Photography, RIT, 1 Lomb Memorial Dr. Rochester, N.Y. 14623
Fourth Internat. Symp. on Ultrasonic Imaging and Tissue Characterization, NBS, Gaithersburg M. Linzer, $R m$. A366, Materials Bldg., NBS, Washington, D.C. 20234

18-21 USNC/URSI/IEEE, mtg., Seattle A. Ishimaru, Dept. of Electrical Eng., FT-10, U. of Washington, Seattle, Wash. 98195

18-22 Laser '79 Exhibition and Congress, West Germany $C$ Werner, DFVLR, Inst. für Physik der Atmosphare, 801 Oberpfaffenhofen, Post Wessling/OBB, German Federal Republic

18-22 Carbon Dioxide Lasers course, Los Alamos Laser Inst. of Am., P.O. Box 9000, Waco, Tex. 76710

18-22 Infrared Technology: Fundamentals and Systems Applications course, U. of Mich. Eng. Summer Confs., 800 Chrysler Ctr., N. Campus, U. of Mich., Ann Arbor, Mich. 48109 